\title{
Relación entre las habilidades de Pensamiento Crítico y Creativo y el Aprendizaje autónomo en estudiantes de la Facultad de Ciencias Matemáticas
}

\author{
Carlos Alberto Peña Miranda ${ }^{1}$, Elizabeth Cosi Cruz ${ }^{2}$
}

\begin{abstract}
Resumen: El objetivo del presente trabajo de investigación fue determinar si el pensamiento crítico y creativo y las estrategias de aprendizaje se relacionan entre sí en una muestra de estudiantes de las cuatro escuelas que conforman la Facultad de Ciencias Matemáticas de la Universidad Nacional de San Marcos. Se utilizó un diseño descriptivo correlacional con una muestra de 300 estudiantes a quienes se aplicaron dos instrumentos de evaluación: el inventario de pensamiento crítico y creativo de Alberto Acevedo y Marcela Carrera y el inventario de aprendizaje autónomo de Manuel Torres. Estos instrumentos fueron sometidos a los análisis estadísticos correspondientes que determinaron que las pruebas son válidas y confiables. Los resultados indican que hay correlaciones significativas y positivas entre el pensamiento crítico y creativo y el aprendizaje autónomo $(\mathrm{r}=0,80)$ en los estudiantes de la muestra.
\end{abstract}

Palabras clave: pensamiento critico y creativo; aprendizaje autónomo; aprendizaje significativo.

\section{Relationship between the skills of Critical and Creative Thinking and Autonomous Learning in students of the Faculty of Mathematical Sciences}

Abstract: The objective of this research was to determine if critical and creative thinking and learning strategies are related to each other in a sample of students from the four schools that make up the Mathematical Sciences Faculty of the National University of San Marcos. A correlational descriptive design was used with a sample of 300 students to whom two evaluation instruments were applied: the inventory of critical and creative thinking of Alberto Acevedo and Marcela Carrera and the independent learning inventory of Manuel Torres. These instruments were subjected to the corresponding statistical analyzes that determined that the tests are valid and reliable. The results indicate that there are significant and positive correlations between critical and creative thinking and autonomous learning $(r=0.80)$ among the students in the sample.

Keywords: critical and creative thinking; autonomous learning; significant learning.

Recibido: 01/10/2017. Aceptado: 16/12/2017. Publicado online: 31/12/2017

CLos autores. Este artículo es publicado por la Revista PESQUIMAT de la Facultad de Ciencias Matemáticas, Universidad Nacional Mayor de San Marcos. Este es un artículo de acceso abierto, distribuido bajo los términos de la licencia Creative Commons Atribucion-No Comercia-Compartir Igual 4.0 Internacional.(http://creativecommons.org/licenses/by-nc-sa/4.0/) que permite el uso no comercial, distribución y reproducción en cualquier medio, siempre que la obra original sea debidamente citada. Para información, por favor póngase en contacto con revistapesquimat.matematica@unmsm.edu.pe

\footnotetext{
${ }^{1}$ UNMSM, Facultad de Ciencias Matemáticas, e-mail: cpenam@unmsm.edu.pe

${ }^{2}$ Universidad Norbert Wiener, e-mail: elizabeth.cosi@uwiener.edu.pe
} 


\section{Introdución}

La presente investigación hace énfasis a dos aspectos muy importantes con el desarrollo del sistema académico universitario ellos son: el pensamiento critico y creativo y el aprendizaje autónomo.

Empezaremos destacando la importancia el aprendizaje autónomo es relevante cuando el estudiante asume el proceso de aprendizaje con un sentido crítico y creativo. Es decir, desde la criticidad aprende a autorregularse para identificar las fortalezas y debilidades de aprendizaje respecto a las habilidades de aprendizaje, los hábitos de estudio, las estrategias de aprendizaje, la inteligencia emocional, la inteligencia existencial, la inteligencia ética, la inteligencia de las relaciones interpersonales, en fin, todo lo concerniente a habérselas con el entorno y el mundo.

Por otro lado, la generación y el uso de un pensamiento crítico y creativo involucran factores de enseñanza que promueva un aprendizaje autónomo que activan actividades de aprendizaje relacionadas con cosas prácticas para alcanzar la resignificación del conocimiento con base en un conocimiento sensorial que favorece la construcción de un conocimiento que engloba toda la dimensión de la persona en el acto de conocer y producir conceptos (Chica, 2010).

Finalmente, el objetivo de la presente investigación es establecer la relación que existe entre las habilidades de Pensamiento Crítico y Creativo y el Aprendizaje autónomo en estudiantes de la Facultad de Ciencias Matemáticas de la Universidad Nacional Mayor de San Marcos

\section{Metodología}

En la medida que las variables independientes ya han ocurrido y por lo tanto no pueden ser manipuladas por el investigador, el presente estudio es una investigación de tipo no experimental.

Para la selección del diseño de investigación se ha utilizado como base el libro de Hernández, R., Fernández, C. y Baptista, P. [3] titulado "Metodología de la Investigación". Según estos autores el diseño adecuado para esta investigación es de tipo descriptiva correlacional.

En nuestro caso, pretendemos relacionar las habilidades de Pensamiento Crítico y Creativo con el Aprendizaje autónomo en una muestra de estudiantes de la Facultad de Ciencias Matemáticas de la Universidad Nacional Mayor de San Marcos.

La muestra está conformada por 300 alumnos del primer ciclo con edades que fluctúan entre 17 y 29 años de edad, siendo las de mayor porcentaje las edades de 20 años con el 22, \%, 21 con $15,8 \%$ y 22 con el $14,8 \%$.

Para la recolección de datos se ha aplicado la técnica de la encuesta para cada una de las variables a todos los estudiantes seleccionados como muestra de estudio y se ha emplearon los siguientes instrumentos

1) Inventario de Pensamiento Crítico y Creativo, es un instrumento que incluye cinco escalas independientes, Toma de decisiones, Fluidez, Motivación, Control emocional y Originalidad. La prueba está compuesta por un total de 34 ítems, los cuales se presentan en listas con cinco alternativas de respuesta, de los cuales el alumno tendrá que escoger la que considere conveniente:

2) Prueba de Aprendizaje autónomo, es un instrumento de diez escalas independientes que evalúan la forma como los estudiantes estudian y aprenden de manera independiente. Esta prueba ha sido construida en nuestro país y ha sido sometida a todos los procedimientos técnicos para garantizar su validez y confiabilidad. 


\section{Resultados}

El análisis de confiabilidad de la prueba de Pensamiento crítico y creativo, tomando en cuenta los 34 ítems correspondientes a las cinco áreas que la componen indica que todos los ítems son aceptados para conformar dicho inventario, y corresponden a cada una de las escalas asignadas por sus autores. El análisis generalizado de la prueba indica que el alfa de cronbach alcanza un valor de 0,80 lo que podemos considerar como muy bueno.

En lo que se refiere a la validez, se realizó a través del Análisis Factorial Exploratorio, aplicando el método de los componentes principales, la que permite observar que la prueba de Pensamiento crítico y creativo presenta una Medida de Adecuación del Muestreo de Kaiser-Meyer-Olkin que asciende a 0,86; el cual puede clasificarse como adecuado. Además presenta un test de esfericidad de Bartlett cuyo valor denota que la matriz de correlaciones entre áreas es significativa, la varianza explicada del orden del 70,76\%. Estos hallazgos indican que la prueba tiene validez de constructo.

En lo que se refiere al instrumento de Aprendizaje autónomo los análisis psicométricos a los que fue sometido, revelan que los 65 ítems forman parte de dicho inventario y corresponden a cada una de las diez escalas asignadas previamente por su autor. El instrumento es confiable en la medida que sus escalas obtuvieron coeficientes Alfa de Cronbach con valores que oscilan entre 0,60 y 0,81, superando ampliamente el criterio de 0,50 y un alfa de Cronbach total de 0,93.

En cuanto a la validez, el resultado del Análisis Factorial Exploratorio indica que la prueba está conformada por un solo factor que explica el 74,02\% de la varianza total. Alcanza además un valor de 0,92 en la medida de adecuación del muestreo de Kaiser-Meyer-Olkin, el mismo que puede considerarse como adecuado. Por consecuencia de los resultados, estos permiten concluir que el instrumento de aprendizaje autónomo presenta confiabilidad y validez de constructo.

\subsection{Análisis correlacionales}

A continuación se muestran los resultados del estudio de correlación realizado mediante el coeficiente de correlación de Spearman entre el Pensamiento Crítico y Creativo y el Aprendizaje Autónomo.

Tabla 1. Análisis de Correlación entre el Pensamiento Crítico y Creativo y el Aprendizaje autónomo

\begin{tabular}{|c|c|}
\hline Variables & Aprendizaje autónomo \\
\hline Pensamiento Crítico y Creativo & $0,80^{* * *}$ \\
\hline
\end{tabular}

Los resultados presentados en la Tabla 1 nos indican que existen correlaciones significativas entre las variables en estudio $(\mathrm{r}=0,80)$.

Estos resultados no hacen sino ratificar que cada persona aprende y se desarrolla de manera distinta y a un ritmo diferente que otros estudiantes, se aplica o se experimenta el aprendizaje en la misma realidad, es muy importante desarrollar un aprendizaje autónomo dependiendo de las habilidades de pensamiento crítico y creativo que haya podido desarrollar a lo largo de su vida. 
Tabla 2. Análisis de Correlación entre las dimensiones del Pensamiento Crítico y Creativo y el Aprendizaje autónomo

\begin{tabular}{|l|c|}
\hline Dimensiones & Aprendizaje autónomo \\
\hline Toma de decisiones & $0,68^{* * *}$ \\
\hline Fluidez & $0,64^{* * *}$ \\
\hline Motivación & $0,66^{* * *}$ \\
\hline Control emocional & $0,62^{* * *}$ \\
\hline Originalidad & $0,74^{* * *}$ \\
\hline
\end{tabular}

$$
\begin{aligned}
& * \mathrm{p}<0,05 * * \mathrm{p}<0,01 * * * \mathrm{p}<0,001 \\
& \mathrm{~N}=300
\end{aligned}
$$

Los resultados presentados en la Tabla 2 nos indican que existen correlaciones significativas entre las dimensiones del Pensamiento Critico y creativo y el Aprendizaje autónomo, notándose que la correlación que alcanzan mayor valor es la dimensión originalidad $(\mathrm{r}=0,74)$ con el total de Aprendizaje Autónomo, esto confirmaría que la originalidad es importante para el aprendizaje autónomo de los estudiantes ya que permite enfrentar la vida con una perspectiva más amplia.

\section{Conclusiones}

Los resultados obtenidos permiten concluir que existe una relación significativa entre las habilidades de Pensamiento Crítico y Creativo y el Aprendizaje autónomo en estudiantes de la Facultad de Ciencias Matemáticas de la Universidad Nacional Mayor de San Marcos.

Finalmente, el análisis de correlación nos muestran que las dimensiones del Pensamiento Crítico y Creativo (toma de dimension, fuidez, motivación, control emocional, y original) se encuentran relacionadas significativamente con el Aprendizaje autónomo.

\section{Referencias bibliográficas}

[1] Campirán, A. (1999). Habilidades de Pensamiento Crítico y Creativo. México: Colección Hiper-COL.

[2] Chica, F (2010). Factores de la enseñanza que favorecen el aprendizaje autónomo en torno a las actividades de aprendizaje. Reflexiones Teológicas, 6 (1),167-195.

[3] Hernández, R., Fernández, C. y Baptista, P. (2006). Metodología de la Investigación. México: Mc Graw Hill

[4] López, M. (2000). Pensamiento crítico y creatividad en el aula. México: Ed. Trillas.

[5] Negrete Fuentes, J. (2007). Estrategias de aprendizaje. México: Limusa.

[6] Siegel, S y Castellan, N.J. (1995).Estadística no paramétrica aplicada a las Ciencias de la Conducta (4a ed.). México: Trillas. 